\title{
Identification of Acyl-CoA Thioesterase in Mouse Mesenteric Lymph Nodes
}

\author{
Takayuki Ohtomo, ${ }^{a}$ Chisato Nakao, ${ }^{a}$ Megumi Sumiya, ${ }^{a}$ Osamu Kaminuma, ${ }^{b}$ Akemi Abe, ${ }^{c}$ \\ Akio Mori, ${ }^{c}$ Niro Inaba, ${ }^{d}$ Tetsuta Kato, ${ }^{e}$ and Junji Yamada ${ }^{*, a}$ \\ ${ }^{a}$ Department of Pharmacotherapeutics, Tokyo University of Pharmacy and Life Sciences; ${ }^{d}$ Center for Fundamental \\ Laboratory Education, Tokyo University of Pharmacy and Life Sciences; ${ }^{e}$ Center for the Advancement of \\ Pharmaceutical Education, Tokyo University of Pharmacy and Life Sciences; Tokyo 192-0392, Japan: ${ }^{b}$ Allergy and \\ Immunology Project, Tokyo Metropolitan Institute of Medical Science; 2-1-7 Kamikitazawa, Setagaya-ku, Tokyo \\ 156-8506, Japan: and ${ }^{c}$ National Hospital Organization, Sagamihara National Hospital, Clinical Research Center for \\ Allergy and Rheumatology; 18-1 Sakuradai, Minami-ku, Sagamihara, Kanagawa 252-0392, Japan. \\ Received December 19, 2012; accepted February 20, 2013
}

Acyl-CoA thioesterases (ACOTs) are a group of enzymes that catalyze the hydrolysis of fatty acyl-CoAs to free fatty acids and $\mathrm{CoA}$, with the potential to regulate the intracellular levels of these molecules. In this study, we show that a cytosolic isoform, ACOT7, is expressed at a significant level in the mesenteric lymph nodes (MLNs) of mice. While crude preparations of the mesenteric visceral fat contained significant levels of palmitoyl-CoA thioesterase activity, enzyme activity was concentrated 6.9-fold in MLNs compared with the residual adipose portion after excision of MLNs. When MLN homogenates were centrifuged, $82 \%$ of the enzyme activity was recovered in the cytosolic fraction, concomitant with almost exclusive recovery of ACOT7. Immunoprecipitation using anti-ACOT7 antibody estimated that $87 \%$ of enzyme activity in the homogenates was accounted for by ACOT7. On MLN sections, the germinal centers of secondary lymphoid follicles were immunostained with the antibody. In MLNs of mice fasted for $16 \mathrm{~h}$, ACOT7 levels were induced 1.8-fold, which reflected a 1.5 -fold increase in enzyme activity. These findings suggest that ACOT7 may be involved in dietary intake-associated responses in fatty acid metabolism in MLNs.

Key words acyl-CoA thioesterase; acyl-CoA thioesterase 7; mesenteric lymph node; visceral fat

Acyl-CoA thioesterases (ACOTs) comprise a group of enzymes that catalyze the hydrolysis of fatty acyl-CoA thioesters to corresponding free fatty acids and CoA. To date, 15 mammalian genes have been identified as ACOT family members. ${ }^{1-4)}$ While ACOTs have the potential to regulate the intracellular levels of acyl-CoAs, free fatty acids, and CoA, their physiological functions are not fully understood. However, recent gene-knockout studies on a subgroup of ACOTs revealed that they profoundly affect lipid homeostasis in association with fatty liver development, obesity, and insulin resistance. $^{5-7)}$ Of the ACOT family members, ACOT1 (cytosolic), ACOT2 (mitochondrial), and ACOT7 (cytosolic) have been well characterized for their enzymatic properties (see refs. 1 and 3 for a review). These ACOTs share a common feature in that their expression is markedly induced by ligands for peroxisome proliferator-activated receptor $\alpha$ (PPAR $\alpha$ ), a nuclear receptor that regulates lipid metabolism-related genes, including the fibrate-class of hypolipidemic drugs. Moreover, ACOT1 and ACOT2 have been implicated in fatty acid catabolism, as emphasized by their upregulation in the liver and heart of fasted or high-fat diet-fed animals. ${ }^{8-11)}$ However, ACOT7 has enzyme activity two orders of magnitude higher than ACOT1 and ACOT2, and its expression is prominent in neurons of the central and peripheral nervous systems. ${ }^{12-14)}$ Thus, ACOT7 may have a role in cellular events other than energy metabolism, as the overexpression of ACOT7 in a macrophage cell line modified the production of prostaglandins. ${ }^{15)}$ Recently, we examined the expression of ACOTs in fat tissues of rats ${ }^{16,17)}$ and unexpectedly found a significant level of acyl-CoA thioesterase activity in the mesenteric lymph

The authors declare no conflict of interest. nodes (MLNs) that contaminated visceral fat preparations. Here, we report that ACOT7 is expressed in mouse MLNs and is responsible for the majority of acyl-CoA thioesterase activity present in this immune tissue.

\section{MATERIALS AND METHODS}

Antibodies Rabbit polyclonal antibodies against ACOT1 and ACOT7 were generated in-house ${ }^{12)}$ and have been previously described. ${ }^{8,11-13,16)}$ It should be noted that the antiACOT1 antibody cross-reacts with ACOT2. Immunoglobulin (Ig) $\mathrm{G}$ was prepared from antisera using protein A sepharose (GE Healthcare, Buckinghamshire, U.K.). Other antibodies were obtained from commercial sources as follows: R-Phycoerythrin (PE)-conjugated rat anti-CD45R/B200 (BD Pharmingen, San Jose, CA, U.S.A.); mouse anti-follicular dendritic cell (FDC) (IgM) (Agilent Technologies, Santa Clara, CA, U.S.A.); mouse anti- $\alpha$-tubulin (Sigma-Aldrich, St. Louis, MO, U.S.A.); mouse anti- $\beta$-actin (Gene Tex, Irvine, CA, U.S.A.); fluorescein (FITC)-conjugated sheep anti-rabbit IgG (Sigma-Aldrich); Alexa Flour 647-conjugated goat anti-mouse IgM (Life Technologies, Carlsbad, CA, U.S.A.); and goat anti-mouse IgA+ IgG+ IgM (KPL, Gaithersburg, MD, U.S.A.).

Animals Nine-week-old male ICR mice were obtained from Tokyo Laboratory Animals Science (Tokyo, Japan) and allowed to acclimatize for 1 week on a diet of standard laboratory chow. Some mice were fasted for $16 \mathrm{~h}$ with free access to water. All animals were treated according to the Guide for the Care and Use of Laboratory Animals published by the US National Institutes of Health (NIH Publication No. 85-23, revised 1996). The study protocol was approved by the Committee of Animal Use and Welfare of Tokyo University of Pharmacy 
and Life Sciences.

Tissue Preparation Tissue homogenates were prepared in SETP buffer comprising $250 \mathrm{~mm}$ sucrose, $1 \mathrm{~mm}$ ethylenediaminetetraacetic acid (EDTA), $10 \mathrm{~mm}$ Tris- $\mathrm{HCl}$ ( $\mathrm{pH} 7.5)$, and Complete protease inhibitor cocktail (Roche Applied Science, Mannheim, Germany) using a Potter-Elvehjem glass homogenizer with a Teflon pestle. For adipose tissues, homogenates prepared at a concentration of $30 \%(\mathrm{w} / \mathrm{v})$ were centrifuged at $15000 \times \boldsymbol{g}$ for $30 \mathrm{~min}$ at $4^{\circ} \mathrm{C}$, and the upper clear oil phase and the fat cake firmly packed at the interface were removed on ice. Subsequently, the lower aqueous phase was re-homogenized and used for analysis. For subcellular fractionation, the MLN homogenates $(1 \mathrm{~mL})$ were centrifuged at $400 \times \boldsymbol{g}$ for $10 \mathrm{~min}$, and the pellets washed once with SETP buffer. The wash and supernatant were combined, centrifuged at $25000 \times \mathbf{g}$ for $10 \mathrm{~min}$, and the resulting supernatant was further centrifuged at $100000 \times \boldsymbol{g}$ for $60 \mathrm{~min}$. The $400 \times \boldsymbol{g}$ (nuclear fraction), $25000 \times \boldsymbol{g}$ (particulate fraction), $100000 \times \boldsymbol{g}$ (microsomal fraction) pellets, and the $100000 \times \mathbf{g}$ supernatant (cytosolic fraction) were suspended in SETP buffer and adjusted to $1-\mathrm{mL}$ volumes, the starting volume of the homogenates. The protein concentrations were determined using a DC protein assay kit (Bio-Rad, Hercules, CA, U.S.A.) with bovine serum albumin as the standard. For immunohistochemistry, mice were anesthetized and perfused via the left ventricle with $4 \%(\mathrm{w} / \mathrm{v})$ paraformaldehyde as described previously. ${ }^{13)}$ The MLNs were excised and processed to paraffin-embedded 3- $\mu$ m-thick serial sections, which were placed on poly-L-lysine-coated glass slides.

Enzyme Assay ACOT activity was measured by spectrophotometry with $20 \mu \mathrm{m}$ palmitoyl-CoA as the substrate and expressed as units (U) of $\mu \mathrm{mol}$ of acyl-CoA hydrolyzed $/ \mathrm{min}$ at $30^{\circ} \mathrm{C}$ as described previously. ${ }^{12)}$

Immunoblotting Proteins were resolved in $10 \%$ sodium dodecyl sulfate (SDS)-polyacrylamide gels and immunoreactivity was detected using ECL advance Western blotting detection reagents (GE Healthcare) as described previously. ${ }^{11)}$ For quantitative analyses, the signal intensities of the bands detected on the membranes were measured and transformed into relative values using a calibration curve generated with known amounts of protein.

Immunoprecipitation A fixed amount of MLN homogenates were incubated at room temperature for $60 \mathrm{~min}$ with increasing amounts of $\mathrm{IgG}$, to which protein A sepharose (GE Healthcare) was added and incubated for another $30 \mathrm{~min}$. These mixtures were centrifuged at $200 \times \boldsymbol{g}$ for $5 \mathrm{~min}$, and then palmitoyl-CoA thioesterase activities were measured in the supernatants. The homogenates were frozen and thawed once before use. Even in the absence of $\mathrm{IgG}, 5 \%$ of enzyme activity found in the homogenates was reduced by centrifugation. Therefore, the initial point of immunoprecipitation was corrected for this 5\% loss of enzyme activity and set at $100 \%$.

Immunohistochemistry Immunohistochemical staining was performed as described previously. ${ }^{13,16)}$ After deparaffinization, serial sections were treated with methanol-hydrogen peroxide, blocked with skim milk, and incubated with a primary antibody followed by a biotinylated secondary antibody using a Vectastain Elite ABC standard kit (Vector Laboratories, Burlingame, CA, U.S.A.). Immunoreactivity was visualized by the peroxidase reaction with $3,3^{\prime}$-diaminobenzidine as the substrate, and the sections were counterstained with methyl green. For double-labeling immunofluorescence, the deparaffinized sections were blocked with skim milk plus anti-mouse $\operatorname{Ig} \mathrm{A}+\mathrm{IgG}+\operatorname{IgM}$ antibody, and sequentially treated with rabbit anti-ACOT7 antibody for $1 \mathrm{~h}$, FITC-conjugated anti-rabbit IgG antibody for $1 \mathrm{~h}$, then PE-conjugated antiCD45R/B200 antibody for $1 \mathrm{~h}$, or mouse anti-FDC antibody (IgM) for $1 \mathrm{~h}$ followed by Alexa Flour 647-conjugated antimouse IgM antibody for $1 \mathrm{~h}$. Sections were examined using a BZ-8100 microscope (Keyence, Osaka, Japan).

Statistical Analysis The statistical significance of differences among values was estimated by using the Mann-Whitney $U$-test or Student's $t$-test. The level of significance was $p<0.05$. Statistical analyses were performed with Prism 4.0 (GraphPad, La Jolla, CA, U.S.A.).

\section{RESULTS}

Acyl-CoA Thioesterase Activity and ACOTs in MLNs Fat pads around the mesentery of mice were excised, and MLNs embedded within the fat pads were carefully separated from adipose tissue. These tissues were homogenized and assessed for palmitoyl-CoA thioesterase activity (Fig. 1A). With whole fat-pad homogenates, $6.8 \pm 0.3 \mathrm{mU} / \mathrm{mg}$ protein of enzyme activity was detected, which was notable since the corresponding values of subcutaneous, retroperitoneal, or epididymal fat pads were less than $50 \%$ of that (data not shown). The enzyme activity of MLNs $(25.4 \pm 1.2 \mathrm{mU} / \mathrm{mg}$ protein) was 6.9 -fold higher than in adipose tissue $(3.7 \pm 1.8 \mathrm{mU} / \mathrm{mg}$ protein). Similar results were obtained in expression levels of ACOT7 estimated by immunoblotting (Fig. 1A, middle panel). Unlike ACOT1 and ACOT2, much higher levels of ACOT7 were detected in the MLNs compared with adipose tissue, and correlated with enzyme activities described above.

The positive correlation between palmitoyl-CoA thioesterase activity and ACOT7 levels was confirmed by subcellular fractionation of MLN homogenates (Fig. 1B). After centrifugation of homogenates, enzyme activity was highly concentrated in the cytosolic fraction and corresponded to $82 \%$ of the sum of enzyme activity recovered in all fractions. We estimated that the recovery of enzyme activity was $77 \%$, based on values from MLN homogenates. It is likely that inactivation and loss of enzyme occurred during handling of the enzyme preparations. However, the corresponding value for the protein was $93 \%$. Under these conditions, ACOT7 was almost exclusively recovered in the cytosolic fraction, unlike ACOT1 and ACOT2 (Fig. 1B, upper and middle panels), suggesting that the enzyme activity found in the MLNs was at least in part due to ACOT7.

Therefore, we performed immunoprecipitation in the MLN homogenates (Fig. 1C). With saturating amounts of antiACOT7 IgG, $87 \pm 5 \%$ (mean \pm S.E.M. of triplicate assays) of the palmitoyl-CoA thioesterase activity was precipitated, whereas $101 \pm 9 \%$ and $102 \pm 19 \%$ of the enzyme activity remained after incubation with anti-ACOT1 $\mathrm{IgG}$ and control $\mathrm{IgG}$, respectively. The source of approximately $10 \%$ of enzyme activity not precipitated by anti-ACOT7 IgG was unclear, although other ACOTs and carboxylesterases can hydrolyze palmitoylCoA. Together, these results indicated that a significant level of acyl-CoA thioesterase activity was present in the MLNs of mice, and was mostly attributable to cytosolic ACOT7 found in this tissue. 
A

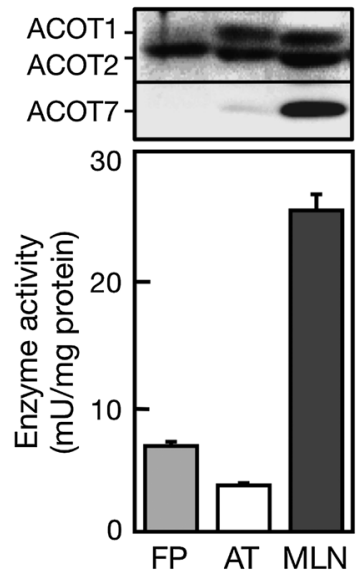

$\mathrm{B}$

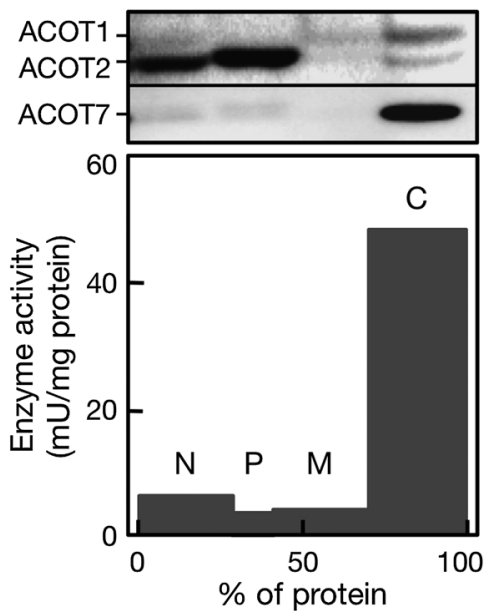

C

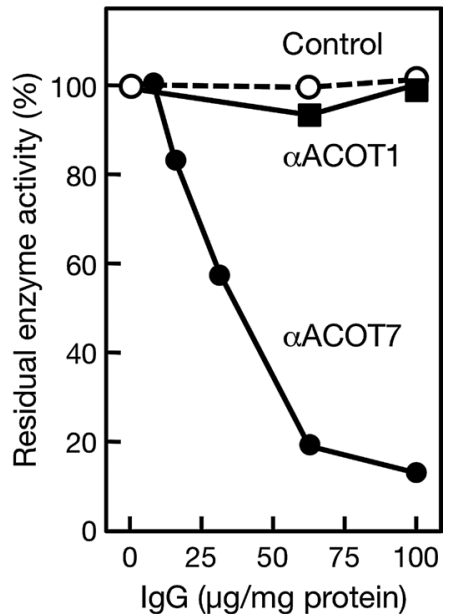

Fig. 1. Acyl-CoA Thioesterase Activity and ACOTs in Mesenteric Lymph Nodes (MLNs)

(A) Fat pads spreading around the mesentery (FP) were excised from mice and MLNs embedded in the fat were carefully isolated from residual adipose tissue (AT). These tissues were homogenized and subjected to enzyme assay for palmitoyl-CoA thioesterase activity (mean \pm S.E.M. of more than three mice), and immunoblotting with anti-ACOT1 or anti-ACOT7 antibody ( $40 \mu \mathrm{g}$ protein/lane). The positions of ACOT1 (46 kDa), ACOT2 (45 kDa), and ACOT7 (43 kDa) are indicated. It should be noted that the anti-ACOT1 antibody cross-reacts with ACOT2. (B) Subcellular fractionation. MLN homogenates were fractionated by sequential centrifugation and subjected to enzyme assay and immunoblotting ( $40 \mu \mathrm{g}$ protein/lane) as described above. N, nuclear; P, particulate; M, microsomal; and C, cytosolic fractions. Recovery after the fractionation was $93 \%$ in protein and $77 \%$ in enzyme activity. MLNs were pooled from ten mice. Results from one of two independent experiments are presented. (C) Immunoprecipitation. MLN homogenates were incubated with increasing amounts of anti-ACOT1 IgG (ם), anti-ACOT7 IgG $(\bullet)$ or control IgG $(\bigcirc)$ prepared from nonimmunized rabbit serum, and then palmitoyl-CoA thioesterase activity was measured in the supernatant obtained by centrifugation. The results are expressed as \% of initial enzyme activity $(20 \mathrm{mU} / \mathrm{mg}$ protein or $40 \mathrm{mU} / \mathrm{tube})$. Each plot represents the mean of measurements in triplicate. MLNs were pooled from ten mice. Results from one of two independent experiments are presented.

The ACOT7 gene generates several isoforms including cytosolic ACOT7a (43 kDa), mitochondrial ACOT7b (43 kDa) and cytosolic ACOT7g $(50 \mathrm{kDa})$ by alternative exon usage ${ }^{18-20)}$ Among them, ACOT7a only was detected in this study, indicated by subcellular fractionation (Fig. 1B, middle panel), where anti-ACOT7 immunoreactivity was undetectable in the particulate fraction (where mitochondrial ACOT2 was observed) and cytosolic fraction except for ACOT7a (43 kDa).

Cellular Distribution of ACOT7 in MLNs MLNs were processed to paraffin-embedded sections and subjected to immunohistochemistry (Figs. 2A-C). With the anti-ACOT7 antibody, relatively large spheroidal structures that corresponded to lymphoid follicles were immunostained within the medulla. Lymphoid follicles are classified as primary and secondary follicles, and the latter is composed of a germinal center and mantle zone. Moreover, primary follicles and the mantle zone of secondary follicles are rich in B-cells (CD45R/B220positive), whereas germinal centers contain follicular dendritic cells (FDCs). ${ }^{21,22)}$ Therefore, double-labeling immunofluorescence was performed with anti-ACOT7 antibody combined with anti-CD45R/B220 (Figs. 2D-F) or anti-FDC antibody (Figs. 2G-I) to differentially delineate lymphoid follicle structures. ACOT7-labeling was observed in the germinal centers of secondary follicles and partially overlapped with FDClabeling (Fig. 2I). However, ACOT7-labeling and CD45R/ B220-labeling did not overlap, suggesting that B-cells do not express ACOT7 although atypical subsets such as B220negative pre-plasma memory B-cells exist ${ }^{23,24)}$ (Fig. 2F). Using antibody against CD4, CD8a, CD11b, CD11c, CD19, F4/80, Gr-1, or I-A[d], the fluorescence labeling did not overlap with ACOT7-labeling (data not shown).

Effect of Fasting on ACOT7 in MLNs As intestinal lymphatics represent the pathway through which absorbed dietary fats are carried into the systemic circulation, MLNs are exposed to high concentrations of lipids at every meal. To examine whether the level of ACOT7 expression in MLNs can be regulated by dietary intake, mice were fasted for $16 \mathrm{~h}$, and MLNs were homogenized and subjected to enzyme assay and immunoblotting (Fig. 3). Palmitoyl-CoA thioesterase activity and ACOT7 levels were increased 1.5- and 1.8-fold, respectively, in MLNs of fasted mice compared with control mice. No changes were observed in ACOT1 and ACOT2 levels (Fig. 3B, left panel). Therefore, of the ACOTs examined, only ACOT7 levels were selectively increased by fasting, reflected by enhanced enzyme activity in MLNs. These results indicated that ACOT7 expression in MLNs responded to dietary intake and decreased its level, although the mechanism for this response was unclear.

\section{DISCUSSION}

This study demonstrated that a significant level of acyl-CoA thioesterase activity was present in mouse MLNs, identified cytosolic ACOT7 as the molecule responsible for this enzyme activity, and localized it to the germinal centers of secondary lymphoid follicles. Moreover, the expression of ACOT7 in MLNs was shown to be affected by dietary intake.

ACOT7 is also known as brain acyl-CoA hydrolase $(\mathrm{BACH})$, with prominent expression in brain neurons. ${ }^{13,14)}$ However, transcriptome analyses revealed abundant expression of ACOT7 in macrophages that could be upregulated by lipopolysaccharide. ${ }^{25)}$ Moreover, these findings were experimentally verified and ACOT7 overexpression was shown to strongly suppress the basal production of prostaglandin D2 and E2 in a macrophage cell line, possibly by restricting incorporation of arachidonic acid into membrane phospholipids. ${ }^{15)}$ Therefore, we focused on FDCs, functionally related to macrophages, as a candidate ACOT7-expressing cell type 

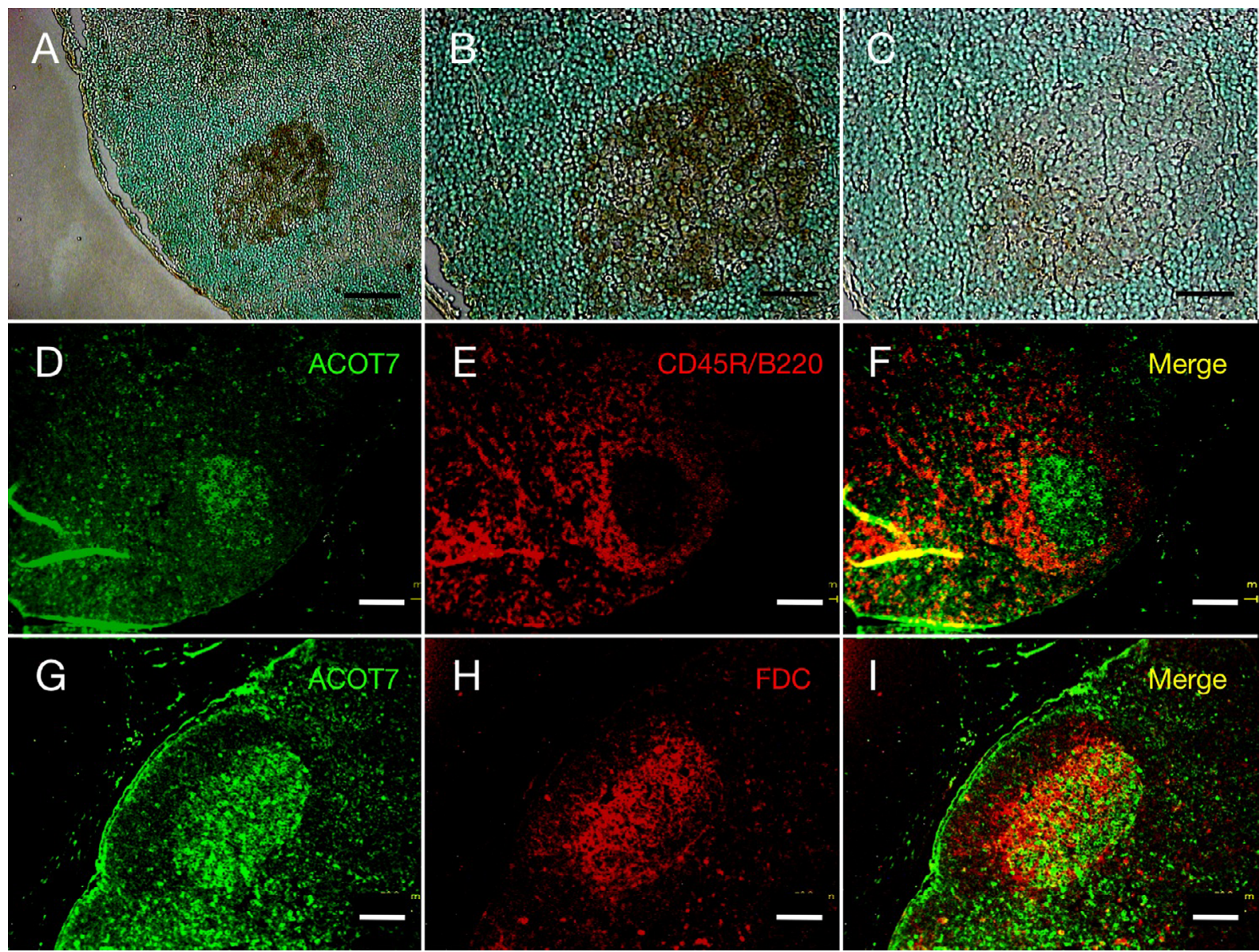

Fig. 2. Immunohistochemistry of ACOT7 in MLNs

Paraffin-embedded MLN sections were immunostained with anti-ACOT7 antibody (A,B). Positive immunoreactivity is shown in brown on the sections counterstained with methyl green. The negative control replaced the primary antibody with non-immunized rabbit serum, in which the IgG concentration was adjusted to the same level as the primary antibody, and a lack of staining was confirmed (C). In addition, double-labeling immunofluorescence was performed with anti-ACOT7 and anti-CD45R/ B220 (D-F) or anti-FDC (G-I) antibodies. The positive immunoreactivities are shown in green for anti-ACOT7 antibody (D, G) and red for anti-CD45R/B220 (E) and anti-FDC (H) antibodies. Merged signals are yellow (F,I). Scale bars: $200 \mu \mathrm{m}$ in A; $100 \mu \mathrm{m}$ in B-I. (Color images were converted into gray scale.)

A

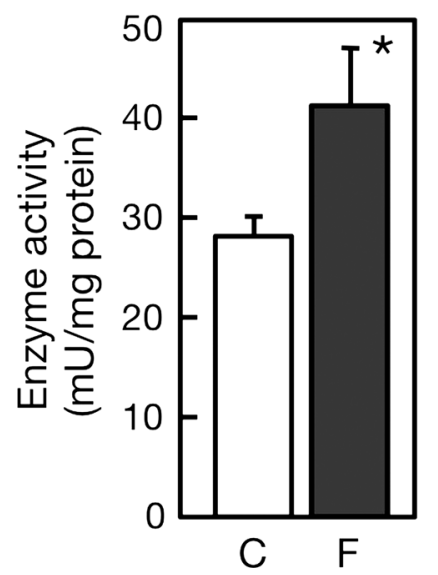

B
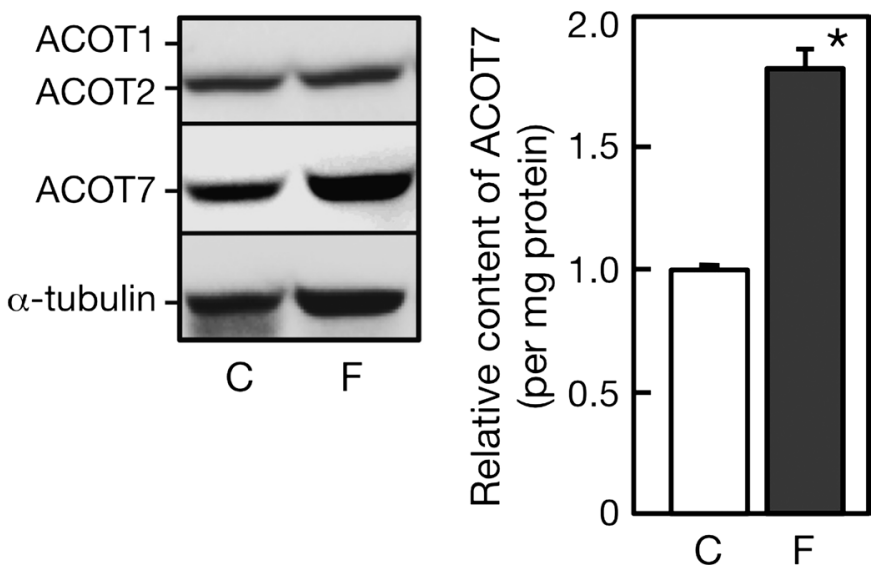

Fig. 3. Effect of Fasting on ACOT7 Expression in MLNs

Mice were fasted for $16 \mathrm{~h}$. MLN homogenates were prepared and subjected to enzyme assay for palmitoyl-CoA thioesterase activity (A), and immunoblotting (20 $\mu \mathrm{g}$ protein/lane) with antibodies against ACOT1, ACOT7, or $\alpha$-tubulin used for loading control (B). Levels of ACOT7 were assessed by quantifying the signal intensities of bands detected on immunoblots, and results are expressed relative to the value for the control, set at 1 . C, control; F, fasted. $* p<0.05$ versus control (mean \pm S.E.M. of five mice).

in the germinal centers of secondary lymphoid follicles. Our results suggested that FDCs are ACOT7-expressing cells. However, we could not determine whether all FDCs expressed ACOT7. The FDC antigen recognized by the antibody used in this study exists on the cell surface (information from manufacturer's certificate) while ACOT7 is cytosolic, making it a little difficult to evaluate whether they were co-expressed in the same cells by double-labeling immunofluorescence. 
Ingested dietary lipids are absorbed and enter the systemic circulation via intestinal lymphatics as chylomicrons. Intestinal lymphatics also represent the main pathway through which toxic mediators disseminate throughout the body. For example, multiple organ dysfunction syndrome, "MODS," secondary to hemorrhagic shock and intestinal ischemia, is mediated by cytokines and lipid mediators produced in the gut and carried in lymph, but not portal venous plasma. ${ }^{26,27)}$ On the other hand, inflammatory cytokines produced in visceral fat deposited around the mesentery and omental are considered causative of systemic insulin resistance observed in obese patients with metabolic syndrome. ${ }^{28-30)}$ If these cytokines are carried in lymph to the circulation, MLNs likely play an important role in the pathophysiology of metabolic syndrome. Indeed, proinflammatory changes originally induced in MLN macrophages by feeding a saturated fat-diet developed to systemic inflammation, in an extreme model of mice. ${ }^{31)}$ Therefore, we propose that ACOT7 may play a role in fat-induced inflammatory responses in MLNs located along the transport pathway to the systemic circulation of taken-up dietary lipids, gut-produced cytokines and lipid mediators. It will be of interest to examine whether ACOT7 can mediate pro- or antiinflammatory responses in MLNs chronically exposed to high fat-containing lymph.

The expression of ACOT7 was not necessarily confined to MLNs among secondary lymphoid tissues. Its expression in axillary lymph nodes was nearly comparable to MLNs, but was barely detectable in inguinal lymph nodes of mice. In the spleen and submandibular lymph nodes, ACOT7 levels were about $30 \%$ or less compared with MLNs (data not shown). Therefore, ACOT7 was preferentially expressed in MLNs among secondary lymphoid tissues.

Acknowledgments This work was supported in part by Grants-in-Aid from the Ministry of Education, Culture, Sports, Science and Technology of Japan, and the Strategic Information and Communications R\&D Promotion Programme (SCOPE).

\section{REFERENCES}

1) Hunt MC, Alexson SE. The role Acyl-CoA thioesterases play in mediating intracellular lipid metabolism. Prog. Lipid Res., 41, 99-130 (2002).

2) Hunt MC, Yamada J, Maltais LJ, Wright MW, Podesta EJ, Alexson SE. A revised nomenclature for mammalian acyl-CoA thioesterases/hydrolases. J. Lipid Res., 46, 2029-2032 (2005).

3) Kirkby B, Roman N, Kobe B, Kellie S, Forwood JK. Functional and structural properties of mammalian acyl-coenzyme A thioesterases. Prog. Lipid Res., 49, 366-377 (2010).

4) Brocker C, Carpenter C, Nebert DW, Vasiliou V. Evolutionary divergence and functions of the human acyl-CoA thioesterase gene (ACOT) family. Hum. Genomics, 4, 411-420 (2010).

5) Kang HW, Niepel MW, Han S, Kawano Y, Cohen DE. Thioesterase superfamily member 2/acyl-CoA thioesterase 13 (Them2/Acot13) regulates hepatic lipid and glucose metabolism. FASEB J., 26, 2209-2221 (2012).

6) Zhang Y, Li Y, Niepel MW, Kawano Y, Han S, Liu S, Marsili A, Larsen PR, Lee CH, Cohen DE. Targeted deletion of thioesterase superfamily member 1 promotes energy expenditure and protects against obesity and insulin resistance. Proc. Natl. Acad. Sci. U.S.A., 109, 5417-5422 (2012).
7) Zhuravleva E, Gut H, Hynx D, Marcellin D, Bleck CK, Genoud C, Cron P, Keusch JJ, Dummler B, Esposti MD, Hemmings BA. Acyl coenzyme A thioesterase Them5/Acot15 is involved in cardiolipin remodeling and fatty liver development. Mol. Cell. Biol., 32, 26852697 (2012).

8) Yamada J, Kuramochi Y, Takoda Y, Takagi M, Suga T. Hepatic induction of mitochondrial and cytosolic acyl-coenzyme a hydrolases/thioesterases in rats under conditions of diabetes and fasting. Metabolism, 52, 1527-1529 (2003).

9) King KL, Young ME, Kerner J, Huang H, O'Shea KM, Alexson SE, Hoppel CL, Stanley WC. Diabetes or peroxisome proliferator-activated receptor alpha agonist increases mitochondrial thioesterase I activity in heart. J. Lipid Res., 48, 1511-1517 (2007).

10) Cole MA, Murray AJ, Cochlin LE, Heather LC, McAleese S, Knight NS, Sutton E, Jamil AA, Parassol N, Clarke K. A high fat diet increases mitochondrial fatty acid oxidation and uncoupling to decrease efficiency in rat heart. Basic Res. Cardiol., 106, 447-457 (2011).

11) Fujita $\mathrm{M}$, Momose A, Ohtomo $\mathrm{T}$, Nishinosono A, Tanonaka $\mathrm{K}$, Toyoda H, Morikawa M, Yamada J. Upregulation of fatty acyl-CoA thioesterases in the heart and skeletal muscle of rats fed a high-fat diet. Biol. Pharm. Bull., 34, 87-91 (2011).

12) Yamada J, Matsumoto I, Furihata T, Sakuma M, Suga T. Purification and properties of long-chain acyl-CoA hydrolases from the liver cytosol of rats treated with peroxisome proliferator. Arch. Biochem. Biophys., 308, 118-125 (1994).

13) Kuramochi Y, Takagi-Sakuma M, Kitahara M, Emori R, Asaba Y, Sakaguchi R, Watanabe T, Kuroda J, Hiratsuka K, Nagae Y, Suga T, Yamada J. Characterization of mouse homolog of brain acyl-CoA hydrolase: molecular cloning and neuronal localization. Brain Res. Mol. Brain Res., 98, 81-92 (2002).

14) Yamada J. Long-chain acyl-CoA hydrolase in the brain. Amino Acids, 28, 273-278 (2005).

15) Forwood JK, Thakur AS, Guncar G, Marfori M, Mouradov D, Meng W, Robinson J, Huber T, Kellie S, Martin JL, Hume DA, Kobe B. Structural basis for recruitment of tandem hotdog domains in acyl-CoA thioesterase 7 and its role in inflammation. Proc. Natl. Acad. Sci. U.S.A., 104, 10382-10387 (2007).

16) Momose A, Fujita M, Ohtomo T, Umemoto N, Tanonaka K, Toyoda $\mathrm{H}$, Morikawa M, Yamada J. Regulated expression of acyl-CoA thioesterases in the differentiation of cultured rat brown adipocytes. Biochem. Biophys. Res. Commun., 404, 74-78 (2011).

17) Ohtomo T, Hoshino A, Yajima M, Tsuchiya A, Momose A, Tanonaka K, Toyoda H, Kato T, Yamada J. Expression and distribution of acyl-CoA thioesterases in the white adipose tissue of rats. Histochem. Cell Biol. (2013), in press.

18) Yamada J, Kuramochi Y, Takagi M, Watanabe T, Suga T. Human brain acyl-CoA hydrolase isoforms encoded by a single gene. Biochem. Biophys. Res. Commun., 299, 49-56 (2002).

19) Takagi M, Kawabe K, Suga T, Yamada J. A 50-kDa isoform of mouse brain acyl-CoA hydrolase: expression and molecular properties. Arch. Biochem. Biophys., 429, 100-105 (2004).

20) Hunt MC, Greene S, Hultenby K, Svensson LT, Engberg S, Alexson SE. Alternative exon usage selectively determines both tissue distribution and subcellular localization of the acyl-CoA thioesterase 7 gene products. Cell. Mol. Life Sci., 64, 1558-1570 (2007).

21) Kasajima-Akatsuka N, Maeda K. Development, maturation and subsequent activation of follicular dendritic cells (FDC): immunohistochemical observation of human fetal and adult lymph nodes. Histochem. Cell Biol., 126, 261-273 (2006).

22) Rebmann S, Gasse H. Bovine lingual tonsil: histomorphological characteristics with special reference to the follicular dendritic cells. Anat. Histol. Embryol., 37, 430-434 (2008).

23) McHeyzer-Williams MG. B cells as effectors. Curr. Opin. Immunol., 15, 354-361 (2003).

24) Shapiro-Shelef M, Lin KI, McHeyzer-Williams LJ, Liao J, McHey- 
zer-Williams MG, Calame K. Blimp-1 is required for the formation of immunoglobulin secreting plasma cells and pre-plasma memory B cells. Immunity, 19, 607-620 (2003).

25) Wells CA, Ravasi $T$, Sultana R, Yagi K, Carninci P, Bono H, Faulkner G, Okazaki Y, Quackenbush J, Hume DA, Lyons PA, RIKEN GER GroupGSL Members. Continued discovery of transcriptional units expressed in cells of the mouse mononuclear phagocyte lineage. Genome Res., 13 (6B), 1360-1365 (2003).

26) Deitch EA. Role of the gut lymphatic system in multiple organ failure. Curr. Opin. Crit. Care, 7, 92-98 (2001).

27) Alexander JS, Ganta VC, Jordan PA, Witte MH. Gastrointestinal lymphatics in health and disease. Pathophysiology, 17, 315-335 (2010).
28) Gregor MF, Hotamisligil GS. Inflammatory mechanisms in obesity. Annu. Rev. Immunol., 29, 415-445 (2011).

29) Sell H, Habich C, Eckel J. Adaptive immunity in obesity and insulin resistance. Nat. Rev. Endocrinol., 8, 709-716 (2012).

30) Romeo GR, Lee J, Shoelson SE. Metabolic syndrome, insulin resistance, and roles of inflammation-mechanisms and therapeutic targets. Arterioscler. Thromb. Vasc. Biol., 32, 1771-1776 (2012).

31) Lichtenstein L, Mattijssen F, de Wit NJ, Georgiadi A, Hooiveld GJ, van der Meer R, He Y, Qi L, Köster A, Tamsma JT, Tan NS, Müller M, Kersten S. Angptl4 protects against severe proinflammatory effects of saturated fat by inhibiting fatty acid uptake into mesenteric lymph node macrophages. Cell Metab., 12, 580-592 (2010). 